\title{
A Scalable Organization of 2-D Ultrasonic Sensor Array
}

\author{
Yuichi Morita*, Yasunobu Takaichi, and Akira Yamawaki \\ Kyushu Institute of Technology, Kitakyushu, 804-8550 Japan \\ *Corresponding Author: yama@ecs.kyutech.ac.jp
}

\begin{abstract}
High resolution 2-D array of ultrasonic sensors needs many cables connecting to the back-end system. This paper proposes a novel front-end structure of 2-D ultrasonic sensor array that needs a single cable, even if many elements are placed in 2-D. In this structure, output from each element is converted to the pulse by an amplifier and a comparator. The 1-bit flip-flop attached to the elements captures this pulse. In 2-D array, column wise flip-flops compose the vertical shift register. Output values from the vertical shift registers are captured by the horizontal shift resister. All outputs of the flip-flops attached to the elements are simultaneously captured into the vertical shift registers. Then, the row of the 2-D array is shifted out to the horizontal shift register. The horizontal shift register shifts out each row value per one clock. As a result, just one cable is needed from the front-end to the back-end without many cables. However, it may take a long time to get all values from all elements because the values are sequentially output per one clock. This time duration may degrade the accuracy of the short range measurement and the frame rate if tracking the moving object. As a preliminary evaluation for the proposed structure, we investigate the relation among resolution, frame rate, and measured distance. We found that the highest resolution in the shortest range measurement can be decided only by the frequency of the speaker and the clock frequency of the shift registers in our analysis. In addition, the frame rate enough to the mobility tracking (60fps) can be achieved by the moderate clock frequency.
\end{abstract}

Keywords: scalable, 2-D array, ultrasonic sensor, time duration, frame-rate, measured distance

\section{Introduction}

2-D arrays of ultrasonic sensors have wider aperture angel and don't need to be moved mechanically than 1-D arrays. So 2-D arrays of ultrasonic sensors make it easier to measure 3-D than 1-D arrays. They are becoming prevalent in many fields; such as Diagnostic Imaging systems in medical (1) and Non Destructive Inspection in maintenance of plants and airplanes ${ }^{(2)}$. As a problem of ultrasonic sensor arrays, the front-end becomes large scale with increase of elements. However, improvement in MEMS technologies enables us to make very small micro-machined ultrasonic sensors. One of them is capacitive micro-machined ultrasonic transducer (CMUT) ${ }^{(3)}$. CMUT enables the frontend to be small with integrated circuits(IC) and many receive elements can be placed. So the high resolution front-end with many ultrasonic sensors is expected ${ }^{(4)}$. But, increase of cables to the back-end that processes a large number of dataset causes to degree improvement of resolution.

In this paper, we will suggest a new front-end structure of 2-D ultrasonic sensor array that has constant cables, even if many elements are placed in 2-D. In this structure, output from each element is converted to the pulse by an amplifier and a comparator. The 1-bit flip-flop attached to the elements captures this pulse. Each element is integrated with flip-flops. So these flip-flops can compose the shift register and values from all elements are sequentially output per one clock. As a result, just one cable is needed from the front-end that reads all values to the back-end.

In this paper, we present how to measure 3-D in our structure. After that, we present the front-end structure. In proposed structure, it may take a long time to get all values from all elements because the values are sequentially output per one clock. This time duration may degrade the frame rate and the accuracy of the short range measurement. As a 

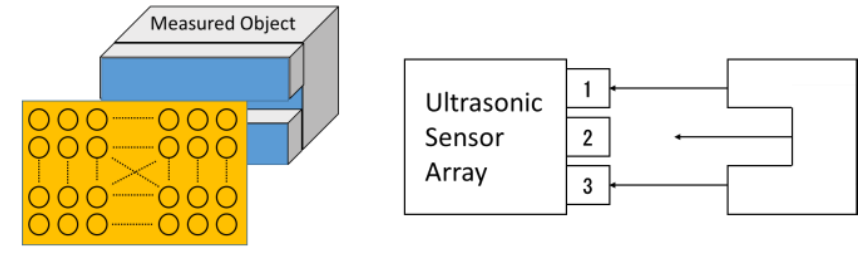

Ultrasonic Sensor Array

Fig. 2.1. Ultrasonic Sensor Array for 3-D Measurement

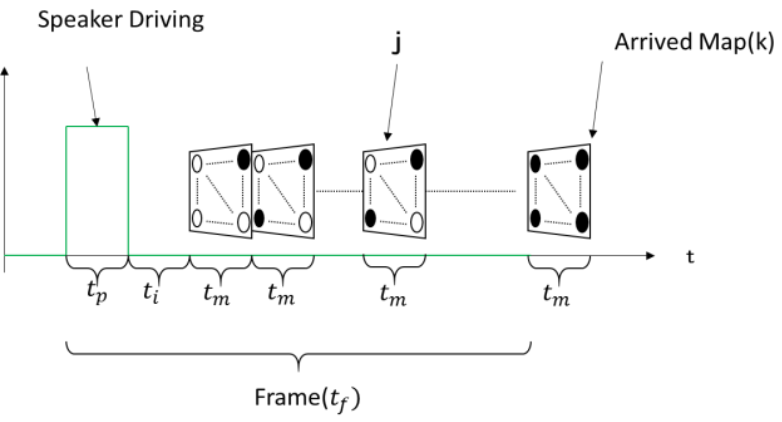

Fig. 2.2. Execution Snapshot of 3-D Measurement

preliminary evaluation for the proposed 2-D ultrasonic sensor array, we will investigate the relation among resolution, frame-rate, and measured distance.

\section{Proposed 2D Sensor Array}

\subsection{Measuring Method on 2-D Ultrasonic Array}

The distance to an object can be measured by the ultrasonic wave. The ultrasonic wave a speaker generates is reflected by the surface of the object. The reflected wave comes back to the ultrasonic sensor placed at the same point of the speaker. The distance from the sensor to the object can be calculated by multipling the traveling time with the sound speed.

As shown in Fig.2.1, a 2-D ultrasonic sensor array can measure the distance between the surfaces of an object and sensors at a time.

As shown in Fig. 2.2, after speakers driving, each cell outputs a binary 2-D map (arrival map) at a certain time. In arrival maps, each bit corresponds to each ultrasonic sensor arranged in 2-D. The " 1 " of bit in the arrival map means that reflected wave has arrived to the sensor. The " 0 " of bit in the arrival map means that reflected wave has never arrived yet.

Some arrival maps are acquired at a certain time interval. We have to consider frame-rates if this organization is applied to real-time 3-D imaging systems. We define $\mathrm{k}$ pieces of arrival maps as one frame. Time to get one frame $t_{f}$ is following expression.

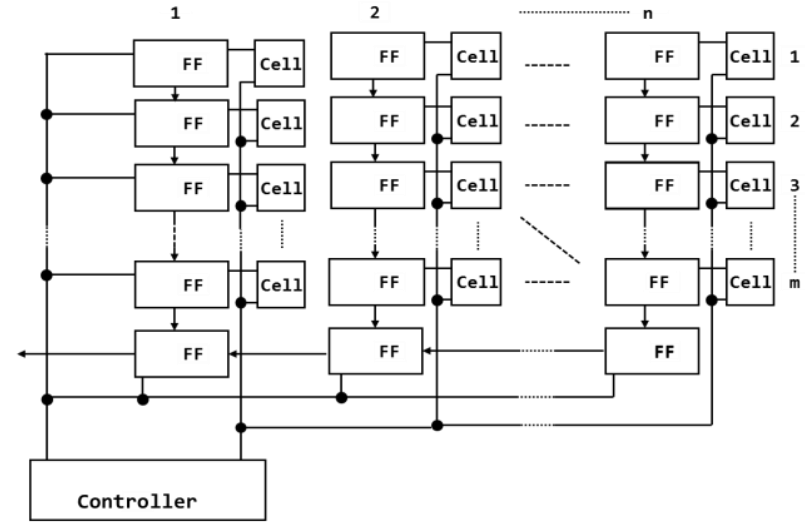

Fig 2.3. Front-End Structure

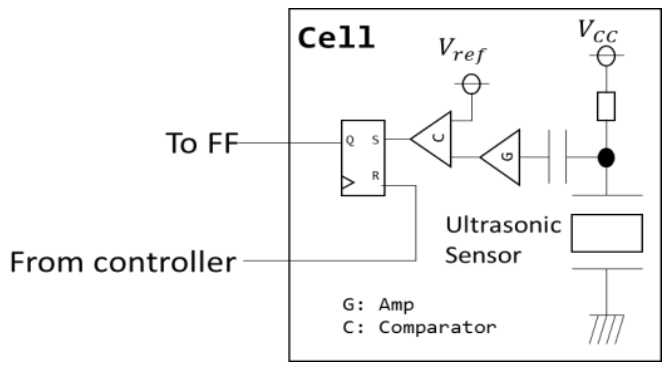

Fig. 2.4. Cell

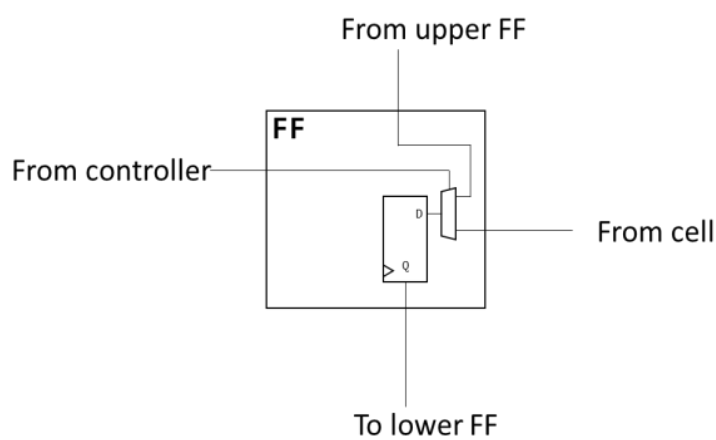

Fig. 2.5. Flip-Flop

$$
t_{f}=t_{p}+t_{i}+k t_{m}
$$

$t_{p}$ is a time driving a speaker. $t_{i}$ is a time duration related to distance to the object and avoiding direct waves. $t_{m}$ is a time to get the whole of each arrival map.

Measured distance $r$ at the arrival map of $j$ can be calculated by following expression.

$$
\mathrm{r}=\frac{t_{i}+(j-1) t_{m}}{2} \mathrm{c}
$$

where $\mathrm{c}$ is sound speed.

\subsection{Front End Organization}

Fig. 2.3 shows the front-end organization consisting of cells, flip-flops, and a controller. Each component is explained as following. 


\section{(a) Cell}

Fig. 2.4 shows the organization of the cell. When waves are transmitted from speakers and reflected in an object, waves are received and converted to voltage by ultrasonic sensors. This voltage is converted to the pulse by an amplifier and a comparator. The 1-bit SR flip-flop captures this pulse. Thus, analog signals from ultrasonic sensors are converted to pulses. Thus other circuits following the cell can be realized by the fully digital circuit.

(b) flip-flops

Fig.2.5 shows the organization of the flip-flop (FF). The FFs are not the buffer to the cell but also they construct the vertical and horizontal shift registers. Except for the FFs placed at the bottom horizontally, signals from the cell and the upper flip-flop are selected by a control signal to the multiplexer. The bottom row of the FFs selects the signal from the upper FF and the right FF. The selected signal is captured into the D flip-flop.

\subsection{Front End Operation}

Let us summarize the operations of the front end as follow.

(1) All SR flip-flops are reset by the controller.

(2) A controller makes speakers transmit ultrasonic waves and wait for a while.

(3) Microphones where ultrasonic waves are reflected in each part arrived set SR flip-flops.

(4) Output values from all SR flip-flops are captured into all vertical shift registers.

(5) Vertical shift registers are shifted out. As a result, values of a line is captured into the horizontal shift register.

(6) The phase (5) is repeated until values of all lines have been shifted out.

(7) An arrived map at a certain time is gotten. If required maps are gotten, this process is finished. If not, the next maps is gotten, returning to (3).

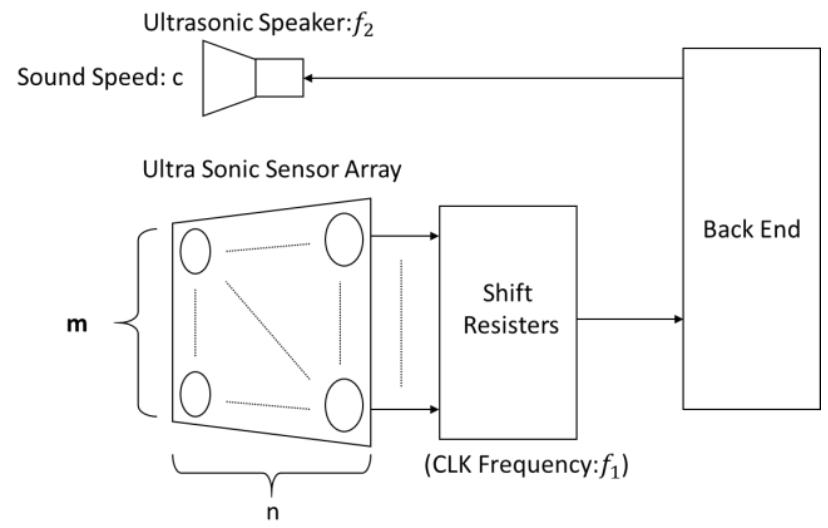

Fig. 3. Assumed System for Evaluation
When the number of cells is $m \times n$ and the CLK frequency of the shift registers is $f$ in Fig. 2.3, a time to get the whole of each arrival map $t_{m}$ can be expressed in following.

$$
t_{m}=\frac{1}{f} \times(m \times n)
$$

\section{Preliminary Evaluation}

\subsection{Evaluation Set up}

Fig. 3 shows the assumed organization of the system with the proposed ultrasonic sensor array. The ultrasonic speaker in the system generates the ultrasonic wave with the frequency, $f 2$. The sound speed is denoted as c. The ultrasonic sensor array and the shift registers are the front end capturing the generated wave from the microphone into the arrival maps. The ultrasonic sensor array has $\mathrm{m} \times \mathrm{n}$ cells. The operational clock (CLK) frequency of the shift registers is $f 1$. The back end controls the ultrasonic microphone and gathers the arrival maps from the front end to generate an ultrasonic 3-D map.

\subsection{Distance resolution}

This system can calculate the distance by using the arrival maps sequentially acquired as shown in Fig. 2.2 and Eq.(2). To get the whole of an arrival map, the CLKs that is the same amount of cells $(n \times m)$ are required. Thus, distance resolution $d_{\min }$ is calculated using the following expression.

$$
\frac{d_{\min }}{c}=\frac{1}{f_{1}} \times(m \times n)
$$

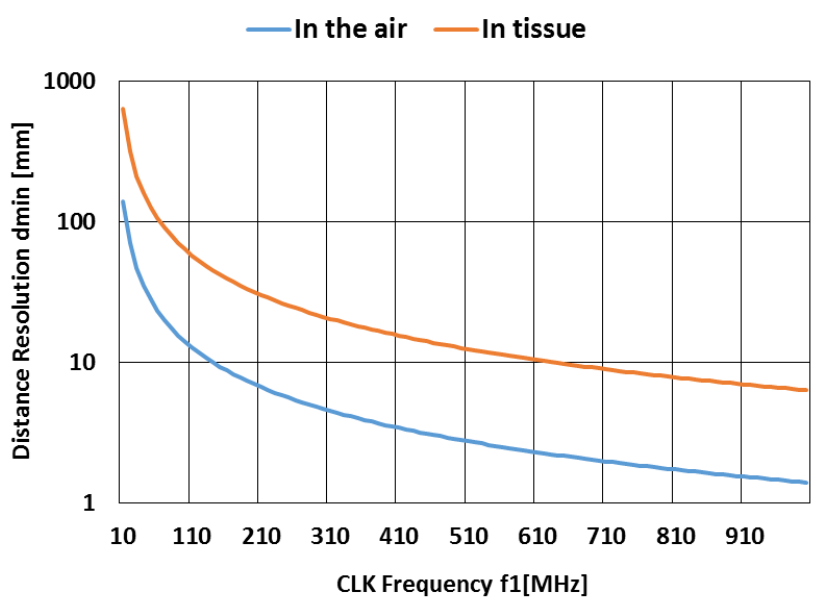

Fig. 3.1. CLK Frequency and Distance Resolution ( $m=64$, $\mathrm{n}=64, \quad \mathrm{~m} \times \mathrm{n}=4096$ ) 


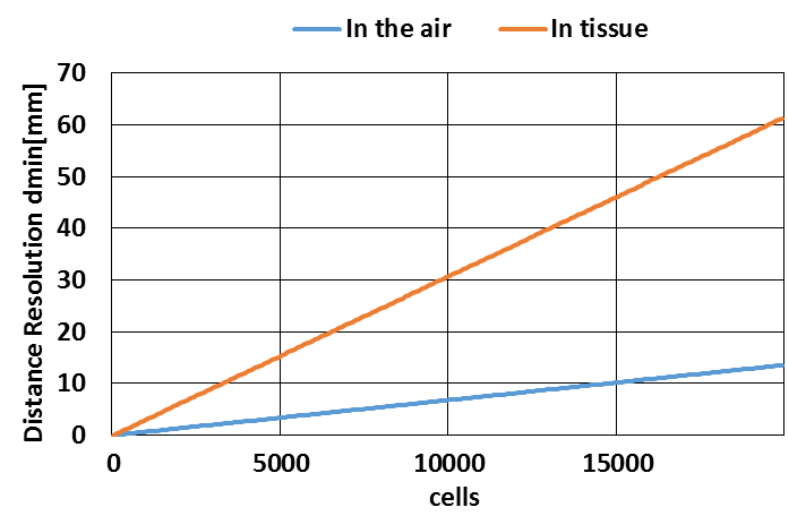

Fig. 3.2. Relation between Cells and Distance Resolution

The $c$ varies according to the material through which the ultrasonic sound passes. For example, the $\mathrm{c}$ is $340 \mathrm{~m} / \mathrm{s}$ in the air and $1540 \mathrm{~m} / \mathrm{s}$ in the tissue.

Fig. 3.1 is relations between CLK frequency $f_{1}$ and distance resolution $d_{\min }$ in $64 \times 64=4096$ cells. Fig. 3.2 is relations between the number of cells $\mathrm{m} \times \mathrm{n}$ and distance resolution $d_{\min }$ in CLK frequency $f_{1}=500 \mathrm{MHz}$. The higher CLK frequency is, the higher distance resolution is, as shown in Fig. 3.1. As show in Fig.3.2, distance resolution has a proportional relation with the number of cells. So the more cells populated, the lower distance resolution is. In the case of a large number of cells are required, CLK frequency must be high to keep high distance resolution.

In addition, the distance that is shorter than the wavelength of the used ultrasonic sound cannot be measured. The wavelength $\lambda$ can be expressed as follow.

$$
\lambda=\frac{c}{f_{2}}
$$

The $40 \mathrm{KHz}$ wavelength in the air is $8.5 \mathrm{~mm}$ and the $5 \mathrm{MHz}$ wavelength in the air is $68 \mu \mathrm{m}$. The $40 \mathrm{KHz}$ wavelength in the tissue is $38.5 \mathrm{~mm}$ and the $5 \mathrm{MHz}$ wavelength in the tissue is $308 \mu \mathrm{m}$. These wavelengths are the inherent limitations of the distance resolution. As making

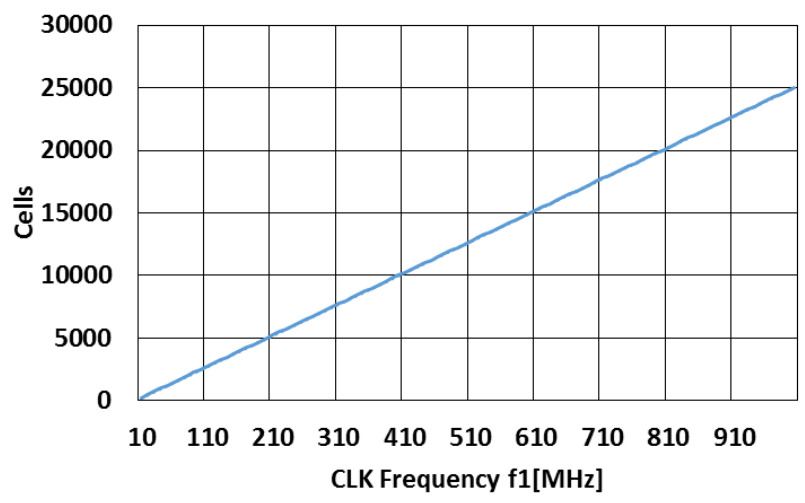

Fig. 3.3. Acceptable Cells on Best Distance Resolution with $40 \mathrm{kHz}$ Ultrasonic

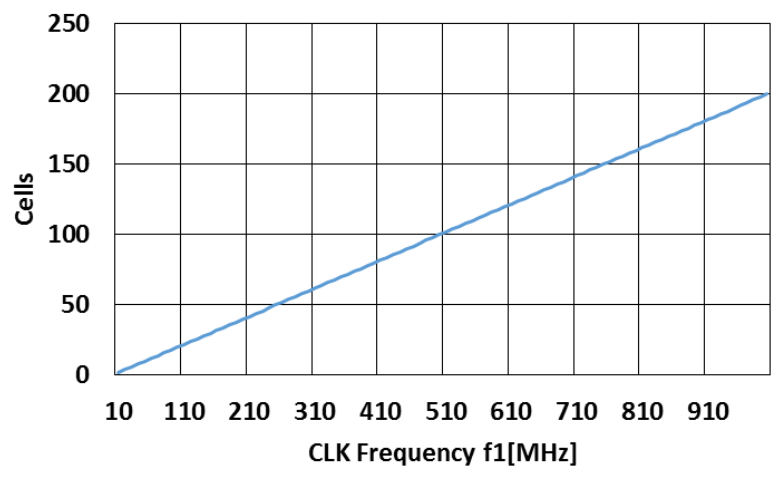

Fig. 3.4. Acceptable Cells on Best Distance Resolution with $5 \mathrm{MHz}$ Ultrasonic

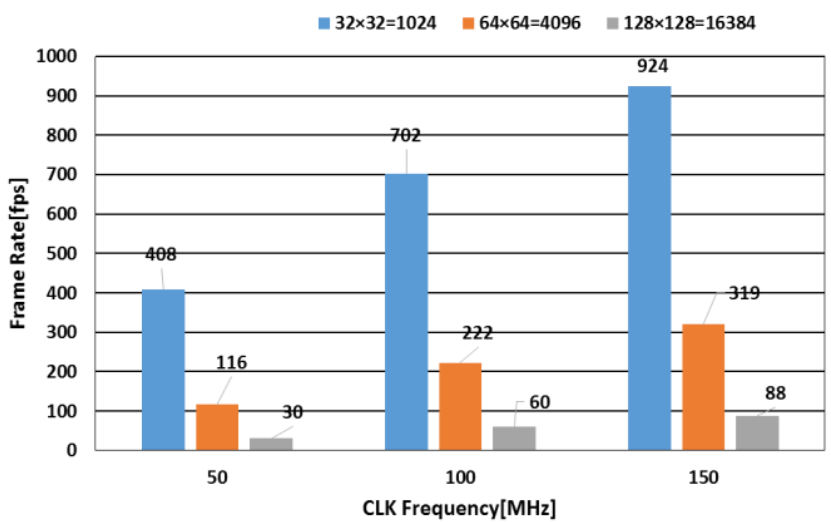

Fig. 3.5 CLK Frequency and Frame Rate $\left(t_{p}=t_{i}=200 \mu s, k=100\right)$

the clock frequency of the shift register higher, the shorter distance resolution can be achieved as shown in Fig.3.1. However, the distance resolution cannot be improved over the limitations due to the wavelength mentioned above.

When the wavelength $\lambda$ is equal with distance resolution, $d_{\text {min }}$, the number of cells is calculated by Eq.(6) and Eq.(7) using Eq.(4).

$$
\frac{\frac{c}{f_{2}}}{c}=\frac{1}{f_{1}} \times(m \times n)
$$

$$
\frac{f_{1}}{f_{2}}=m \times n
$$

Eq. (7) shows the relations among the number of cells, the CLK frequency of the shift registers, and the frequency of the speaker in the max distance resolution. The number of cells doesn't depend on the sound speed $c$ and is expressed by the ratio of the frequency of the shift registers $f_{1}$ to the frequency of the speaker $f_{2}$. Fig. 3.3 and Fig. 3.4 are the relations between CLK frequency and the number of cells. Fig. 3.3 is in $f_{2}=40 \mathrm{KHz}$ and Fig. 3.4 is in $f_{2}=5 \mathrm{MHz}$.If the frequency of the ultrasonic sound is higher, the number of cells can be increased. 


\subsection{Flame Rate}

Considering that the $40 \mathrm{kHz}$ speaker is used in Fig. 3, Fig. 3.5 show the relations between the CLK frequency and the flame rate when $t_{p}$ and $t_{i}$ is $200 \mu \mathrm{s}$ and $\mathrm{k}$ is 100 .

The flame rate decreases with the increase of the cells. However, the higher the CLK frequency of the shift registers is, the higher the frame rate is. So we need to adjust the CLK frequency of the shift registers for the required frame rate and the number of maps and cells.

\section{Conclusions}

We have proposed new front-end structure of 2-D ultrasonic sensor array that needs a single cable to connect the front end and the back end. The proposed front end gets all values sequentially. This time duration may degrade the frame rate and the accuracy of the short range measurement.

As a preliminary evaluation for the proposed 2-D ultrasonic sensor array, we investigated the relation among resolution, frame-rate, and measured distance. As a result, resolution is improved if the CLK frequency of the shift registers is high, although sound speed is different in measured environment. So short range measurement can be done in high resolution. Regarding the frame rate, $60 \mathrm{fps}$ is enough to use usually. Even if the frame rate depends on the number of cells and maps, such rate can be realized by adjusting the CLK frequency of the shift register.

This work is at the stage of a preliminary evaluation. As further work, we will develop a prototype system realizing our proposal shown in this paper. Then, we will perform the detailed evaluations by using the real machine. In addition, we will investigate the real application using our proposal.

\section{References}

(1) Yasutsugu Seo, Keisuke Hashimoto, Ryoichi Kanda, Satoru, Tezuka, Nobuyuki Iwata, Shinichi Hashimoto, Yoichi Ogasawara, Yasuhiko Abe, Hironobu Hongo, and Yasuo Miyajima : "Development of real-time threedimensional ultrasound imaging system", Acoustical Society of Japan, Volume:61, Issue:3, pp.109-117, 2005 in Japanese

(2) Motohisa Abe, Hirokazu Karasawa : "MatrixeyeTM Portable 3D Ultrasonic Inspection System”, TOSHIBA REVIEW, Volume: 60,No.4, pp.48-51, 2005

(3) Ira O. Wygant, Xuefeng Zhuang, David T. Yeh, Omer Oralkan, A. Sanli Ergun, Mustafa Karaman,
Butrus T. Khuri-yakub : "Integration of 2D CMUT arrays with front-end electronics for volumetric ultrasound imaging", IEEE Transactions on Ultrasonic, Ferroelectrics, and Frequency Control, Volume: 55, Issue: 2, pp.327-342, 2008

(4) Anshuman Bhuyan, Jung Woo Choe, Byung Chul Lee, Ira O. Wygant, Amin Nikoozadeh, Ömer Oralkan, Butrus T. Khuri-Yakub, "Integrated Circuits for Volumetric Ultrasound Imaging With 2-D CMUT Arrays", IEEE Transactions on Biomedical Circuits and Systems, Volume: 7, Issue: 6, pp.796 -804, 2013 